\title{
Polymeric endovascular strut and lumen detection algorithm for intracoronary optical coherence tomography images
}

Junedh M. Amrute

Lambros S. Athanasiou

Farhad Rikhtegar

José M. de la Torre Hernández

Tamara García Camarero

Elazer R. Edelman 


\title{
Polymeric endovascular strut and lumen detection algorithm for intracoronary optical coherence tomography images
}

\author{
Junedh M. Amrute, ${ }^{a, b}$ Lambros S. Athanasiou, ${ }^{b, c, \star}$ Farhad Rikhtegar, ${ }^{b}$ José M. de la Torre Hernández, \\ Tamara García Camarero, ${ }^{d}$ and Elazer R. Edelman ${ }^{b, c}$ \\ ${ }^{a}$ California Institute of Technology, Division of Biology and Biological Engineering, Pasadena, California, United States \\ bMassachusetts Institute of Technology, Institute for Medical Engineering and Sciences, Cambridge, Massachusetts, United States \\ 'Brigham and Women's Hospital, Harvard Medical School, Cardiovascular Division, Boston, Massachusetts, United States

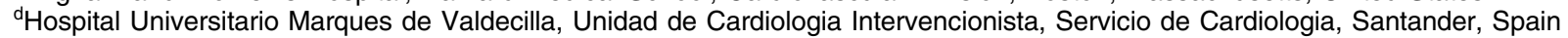

\begin{abstract}
Polymeric endovascular implants are the next step in minimally invasive vascular interventions. As an alternative to traditional metallic drug-eluting stents, these often-erodible scaffolds present opportunities and challenges for patients and clinicians. Theoretically, as they resorb and are absorbed over time, they obviate the long-term complications of permanent implants, but in the short-term visualization and therefore positioning is problematic. Polymeric scaffolds can only be fully imaged using optical coherence tomography (OCT) imaging - they are relatively invisible via angiography-and segmentation of polymeric struts in OCT images is performed manually, a laborious and intractable procedure for large datasets. Traditional lumen detection methods using implant struts as boundary limits fail in images with polymeric implants. Therefore, it is necessary to develop an automated method to detect polymeric struts and luminal borders in OCT images; we present such a fully automated algorithm. Accuracy was validated using expert annotations on 1140 OCT images with a positive predictive value of 0.93 for strut detection and an $R^{2}$ correlation coefficient of 0.94 between detected and expert-annotated lumen areas. The proposed algorithm allows for rapid, accurate, and automated detection of polymeric struts and the luminal border in OCT images. ๑ 2018 Society of Photo-Optical Instrumentation Engineers (SPIE) [DOI: 10.1117/1.JBO.23.3.036010]
\end{abstract}

Keywords: polymeric scaffolds; optical coherence tomography; strut; lumen; bioresorbable vascular scaffold.

Paper 170542RRR received Aug. 15, 2017; accepted for publication Feb. 23, 2018; published online Mar. $20,2018$.

\section{Introduction}

Coronary artery disease (CAD) is a major contributor to death in developed countries and accounts for almost one third of all deaths in individuals over 35 years. ${ }^{1} \mathrm{CAD}$ is characterized by the development of atherosclerosis and occlusive plaque burden in the coronary artery. ${ }^{2,3}$ Arterial stenoses impair distal flow and induce cardiac destruction with myocardial infarction. ${ }^{4}$ Minimally invasive procedures, such as metallic stent and polymeric scaffold angioplasty, address specific lesions ${ }^{5}$ without resorting to open-heart surgery, minimizing procedural risk and speeding recovery times. ${ }^{5,6}$ Endovascular implants are inserted with a catheter through one of the major arteries like the femoral artery and are usually balloon-expanded to hold open a stenosed region, enabling previously constricted blood to pass freely. ${ }^{7}$

While metallic stents remain the most widely used coronary implants, they induce sustained injuries remodeling the coronary artery after waves of endothelial damage, inflammation, tissue proliferation, and disturbed vasomotion. ${ }^{8,9}$ Erodible polymeric implants ${ }^{10}$ allow restoration of normal endothelial function and biomechanical characteristics that may never be recovered with the permanent metallic stents. ${ }^{11-13}$ Polymeric implants are currently in the early stages of development; consequently, there is a scarcity of computational methods to quantify their effects on patient cardiovascular function. In particular,

*Address all correspondence to: Lambros S. Athanasiou, E-mail: lambros@mit .edu bioresorbable vascular scaffolds (BVS) are a commonly used polymeric endovascular implant, and, in spite of their benefits, concern has been raised due to the excess thrombosis and myocardial infarctions associated with BVS. ${ }^{14,15}$ These adverse complications of BVS are a result of the large strut size, which alters the pulsatile arterial flow patterns and leads to arterial thrombosis and myocardial infarctions. Safe use of this technology requires a greater understanding of the basic mechanics of erosion $^{10}$ and interaction with the blood vessel wall. ${ }^{13}$ To achieve this, we need to study the biomechanical effects of polymeric scaffolds on the coronary artery, and this requires accurate three-dimensional (3-D) representation of the struts and luminal border. The first step in such a representation is the development of a robust and fully automated polymeric strut and lumen detection methodology from two-dimensional (2D) intracoronary images.

Optical coherence tomography (OCT) is an intracoronary light-based imaging modality, whose finer resolution of 1 to $15 \mu \mathrm{m}^{16,17}$ allows for detailed evaluation of the luminal border, strut outlines, vessel wall morphology, and plaque characterization. ${ }^{17}$ Given these benefits, OCT is becoming the primary clinical imaging modality used to detect the lumen border and scaffold struts and to estimate plaque composition and volume $^{18,19}$ in the coronary artery. In intracoronary OCT images, polymeric struts and, in particular, BVS struts appear as rectangular

$1083-3668 / 2018 / \$ 25.00$ @ 2018 SPIE 
polygonal structures with well-defined edges and a black core while only the adluminal side of metallic stents can be seen as bright fringes with a dark shadow behind them. ${ }^{20}$ Presently, numerous methods exist to detect metallic stents, ${ }^{21,22}$ which cannot be applied to polymeric scaffold struts given their physical and material differences. ${ }^{23,24}$ Additionally, in images with apposed polymeric struts, traditional lumen detection methods ${ }^{25}$ fail to accurately detect the luminal border.

Despite the emerging popularity of the polymeric scaffold technology, to our knowledge, there is only one semiautomated method to detect BVS struts in OCT images. ${ }^{16}$ This method requires a different algorithm to detect struts above the arterial wall (baseline: right after implantation of the polymeric scaffold) and those embedded inside the tissue (follow-up: few months postimplantation of the polymeric scaffold) and uses percentile-based thresholding for each patient. The user specifies whether the intravascular OCT pullback is baseline or follow-up, making it difficult to detect polymeric struts in patients who have a combination of embedded and apposed struts. Furthermore, a percentile-based thresholding technique is not robust over a large patient population as there is no automated way to find an optimal percentile value for all OCT pullbacks.

To address these limitations, we propose a new methodology that utilizes $K$-means clustering to automatically segment OCT

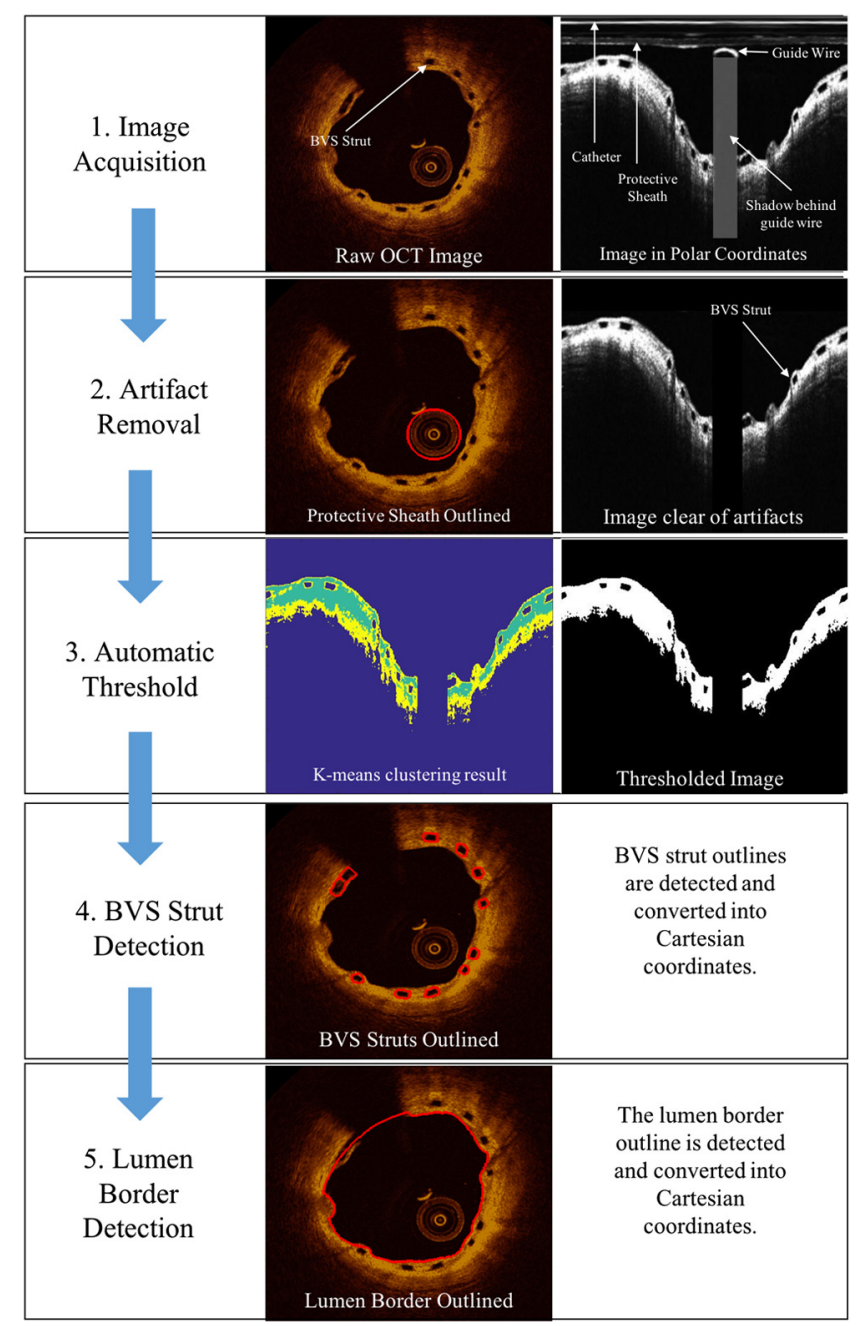

Fig. 1 Overview of the image processing pipeline applied to detect the BVS struts and luminal border in intracoronary OCT images. images. This approach can be adapted to detect polymeric struts with a polygonal definition and well-defined edges. We apply this methodology to detect BVS struts and the luminal border in images with the polymeric implant. The methodology robustly removes imaging artifacts (catheter, protective sheath, and guide wire), thresholds the image, and detects the BVS strut core and true luminal border independent of strut position. The innovative aspects of the proposed methodology include automatic segmentation of OCT images to detect polymeric strut outlines; detection of well-apposed, malapposed, and embedded struts without the need for predefined threshold values; and accurate detection of the luminal border in scaffolded segments.

\section{Materials and Methods}

We use the following steps (Fig. 1): sequential raw OCT image acquisition from patient OCT pullback, preprocessing to denoise the image and remove the guide wire, imaging catheter, and protective sheath, automated segmentation using the $K$-means clustering algorithm, detection of the polygonal shaped polymeric strut core, and detection of the luminal border by removing the detected polymeric struts.

\subsection{Image Acquisition and Preprocessing}

The raw OCT image consists of a sequence of A-lines (columns) in an image matrix, whose values represent the optical energy as a function of time. This image is stored in polar coordinates, where each pixel has intensity $I(r, \theta)$, such that $r$ is the range parameter and $\theta$ is the angle of acquisition, which is converted to Cartesian coordinates to visualize the arterial features, $I(r, \theta) \rightarrow I(x, y)$, where $x=r \cos (\theta)$ and $y=r \sin (\theta)$. The raw OCT image has dimensions $504 \times 952$, giving a total of 479,808 pixels. The image uses a linear scale, and pixel intensity values are normalized between a $[0,1]$ scale. A bilateral filter and edge detection method are applied to reduce background noise and remove artifacts, respectively.

\subsubsection{Bilateral filtering}

A bilateral filter is a nonlinear filter that reduces overall noise in the image while preserving the sharpness of edges. ${ }^{26}$ The intensity value of each pixel in the image matrix is replaced by a weighted average of the neighboring pixels in a predefined neighborhood kernel, $N$. This weight is nonlinear and is sampled from some specified distribution functions. ${ }^{26,27}$ Unlike the Gaussian filter, whose weight is purely dependent on Euclidean distances between pixels, ${ }^{28}$ the bilateral filter uses a weight that is a combination of both Euclidean distances and intensity differences between neighboring pixels. ${ }^{27}$ Integrating both intensity and distance gradients in the smoothing function not only reduces the overall noise in the image but also enhances edge detection. Formally, the bilateral filter is defined as follows:

$I^{f}(\hat{x})=\frac{1}{W_{P}} \sum_{\hat{x}_{i} \in N} I\left(\hat{x}_{i}\right) f_{r}\left[\left\|I\left(\hat{x}_{i}\right)-I(\hat{x})\right\|\right] g_{s}\left(\left\|\hat{x}_{i}-\hat{x}\right\|\right)$,

where $I^{f}$ is the filtered image, $I$ is the raw input image, $\hat{x}$ is the position of the central pixel, $\hat{x}_{i}$ is each pixel in the defined neighborhood $N, f_{r}$ is a Gaussian range filter (which uses intensity gradients), $g_{s}$ is a Gaussian spatial filter (which uses the Euclidean distances between pixels), and the normalization term $W_{P}$ is defined as follows: 
$W_{P}=\sum_{\hat{x}_{i} \in N} f_{r}\left[\left\|I\left(\hat{x}_{i}\right)-I(\hat{x}) \mid\right\|\right] g_{s}\left(\left\|\hat{x}_{i}-\hat{x}\right\|\right)$

to ensure that the total image energy is preserved. ${ }^{26,27}$ In this methodology, we used a $3 \times 3$ kernel window with the smoothing parameter $\sigma_{\mathrm{s}}=1.0$ for both Gaussian kernel functions. Several other kernel dimensions and smoothing parameters were used, but these gave the best results.

\subsubsection{Catheter artifact detection}

To locate the start of the imaging catheter in the OCT image, we find the first pixel in the first and last A-line whose intensity is above 0.6 intensity units. We tried other intensity thresholds; however, in all of the 1140 images, the catheter intensity was $\sim 0.6$ intensity normalized units.

\subsubsection{Protective sheath detection}

Some existing methods ${ }^{29}$ assume that the protective sheath is a straight line located at a fixed distance $(0.45 \mathrm{~mm})$ below the start of the imaging catheter, while other methods use edge detection to find the exact curvature ${ }^{16}$ of the protective sheath. The straight-line approximation holds in most cases but is not robust and leads to problems when the sheath outline is close to or touching the polymeric struts or lumen border. In these cases, this oversimplification of the sheath geometry leads to the deletion of parts of the polymeric struts or lumen border. Therefore, to accurately delete the protective sheath, we use an edge detection method by walking along the gradient of the sheath outline. ${ }^{16}$ To do so, we convert the image matrix to an undirected weighted graph and use Dijkstra's algorithm ${ }^{30}$ to find the minimum cost path.

To construct the cost function to transition between pixels, we use a four-connectivity model ${ }^{16}$ in a $3 \times 3$ neighborhood. Using a $3 \times 3$ kernel window is justified because the sheath geometry is sufficiently simple and uniform. To determine the weights in our cost function, we calculate the inverse of the directional gradients as follows:

$W_{x}=\frac{1}{G_{x}} \quad$ and $\quad W_{y}=\frac{1}{G_{y}}$,

where $W_{x}$ and $W_{y}$ are the weights in the horizontal and vertical directions, respectively, and $G_{x}$ and $G_{y}$ are the intensity gradients in the $x$ and $y$ directions, respectively. Using the inverse gradients to specify the weights implies that the strongest edges in the image have the smallest weights in the graph, and thus walking along the gradient in the image is equivalent to taking the shortest cost path in the graph. For a pixel starting at $(i, j)$, we assign a weight of $W_{x}^{i j}$ for all nodes in the horizontal direction and of $W_{y}^{i j}$ for all nodes in the vertical direction. We construct the graph such that each structure in the graph is defined by the start node, end node, and associated node transition cost [Eq. (3)]. Using this graph, we apply Dijkstra's algorithm to find the minimum cost path between the start and end nodes. ${ }^{30}$

\subsubsection{Guide wire removal}

The region below the guide wires always presents as a black shadow (Fig. 1). The length of the guide wire $(L)$ is approximately $30 \pm 2$ pixels and depends on its position relative to that of the catheter. In our algorithm, we use the average length of $L=30$. To remove the guide wire, we found the A-lines with the smallest sum intensity and removed this region by

1. scanning each column and calculating the total intensity of all pixels in that column as $I_{T}$, where $I_{T}=$ $\left\{I_{1}, I_{2}, \ldots, I_{n}\right\}$ and $I_{i}$ is the sum intensity for Aline $i$,

2. iterating through $I_{T}$ and finding a subset of $L$ consecutive A-lines with a minimum combined sum using the following:

a. From $I_{T}$, we created a vector $S$ of length $n-L$, such that the $i$ 'th element of $S, S_{i}$ is given by $\sum_{j=i}^{i+70} I_{j}$.

b. The starting column of the guide wire corresponds to the index $i$ such that $\min (S)=S_{i}$.

c. The last column of the guide wire is then $i+L$.

3. Deleting the entire region between the $i$ 'th and $i+L$ 'th A-lines.

In cases where the guide wire is smaller than 30 pixels, we delete a small portion of the lumen, and, in cases where the length is greater than 30 pixels, some portion of the guide wire remains unremoved. In either case, the error is only 2 pixels for a $500 \times 952$ image and does not affect our segmentation result.

\subsection{Automated Segmentation}

To segment the image automatically, we use a $K$-means clustering algorithm with $k=3$ clusters $;{ }^{31}[\mathrm{k}=2,3$, and 4 cluster values were tested, but $k=3$ produced the highest positive predictive value (PPV)]. The $K$-means algorithm is an unsupervised machine learning algorithm that takes as an input a set of $N$ observations $\left\{x_{1}, x_{2}, \ldots, x_{N}\right\}$ and classifies them into $k$ clusters $S=\left\{S_{1}, S_{2}, \ldots, S_{k}\right\}$ to minimize the sum of squares residual error within each cluster. We can formally define this clustering problem as the minimization of an objective function:

$\arg \underset{S}{\min } \sum_{i=1}^{k} \sum_{x \in S_{i}}\left\|x-\mu_{i}\right\|^{2}$,

where $\mu_{i}$ is the mean intensity of all the points in cluster $S_{i}{ }^{32} \mathrm{We}$ apply this algorithm to each OCT image in the pullback for $k=$ 3 clusters and produce a masked image (Figs. 1, 3, and 4), where each pixel in the image is classified to one of three clusters. The cluster with the minimum mean intensity is the noise while the remaining clusters contain features of interest, such as polymeric strut and luminal border outlines. To convert this masked image into a binary image, we calculated the mean intensity value of each of the three clusters, and, for all pixels in the minimum mean intensity cluster, we set their intensity value to 0 and all remaining pixels to 1 .

\subsection{BVS Strut Detection}

We use the thresholded image [Fig. 2(d)] to identify the polygonal shaped polymeric strut cores. For each A-line, to classify two points as the start and end of the polymeric strut core, we (1) define the start of the strut core as the pixel, where the intensity drops from 1 to 0 and the end when the intensity jumps from 0 to 1 and (2) only keep the pair of start and end points if the 


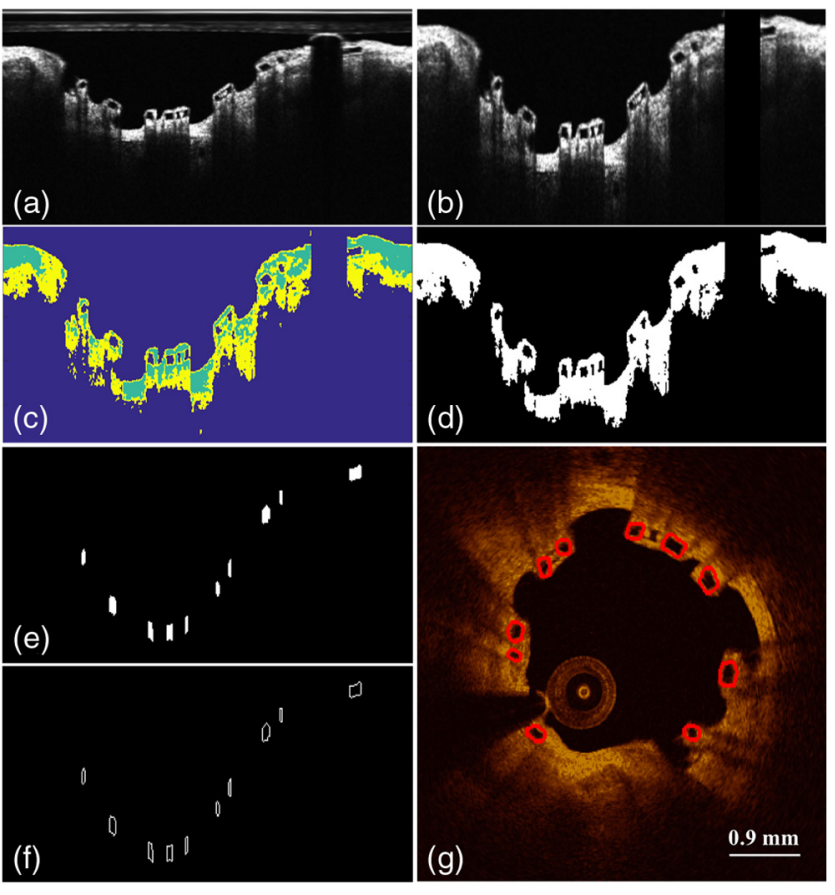

Fig. 2 Automated detection applied to BVS struts: (a) raw OCT image, (b) OCT image cleared of artifacts using the procedure in Sec. 2.2, (c) $K$-means segmentation masked image (with $k=3$ clusters), (d) binary thresholded image, (e) BVS struts segmented as blocks, (f) BVS strut outline, and (g) BVS strut boundaries represented in Cartesian coordinates in red.

distance between them is within a prespecified range (based on typical BVS physical specifications, but this step can be adapted for polymeric struts with other dimensions).

Once we identified the start and end points of the strut core within each A-line, we set all pixels between these points to 1 intensity units and all other pixels to 0 . This produces an image with candidate strut cores [Fig. 2(e)]. To account for the missed strut cores from some A-lines, we use a morphological disk with a radius of 1 to dilate the image. Finally, we removed false positives (FPs) by including only candidates whose aspect ratio is less than 5 and area is between 20 and 40 pixels $^{2}$. Crucially, BVS struts commonly fracture and will resorb ${ }^{10}$ over time and, consequently, do not have a fixed shape or size; ${ }^{33}$ to account for this variability, we accept candidates within a prespecified range. We then use a perimeter function to find the outline of the strut cores [Fig. 2(f)] and then convert the image to Cartesian coordinates [Fig. 2(g)].

\subsection{Lumen Border Detection}

Current methods ${ }^{25}$ used to detect the lumen border fail in the presence of well-apposed polymeric struts because the struts obscure the true lumen border. Consequently, to detect an approximation of the lumen border in images with well apposed polygonal shaped polymeric struts, we process the image by (Fig. 3) deleting the entire region behind each strut, applying the $k$-means algorithm with $k=2$ clusters, converting the image into a binary image using the following rule: setting the cluster with the lowest mean intensity to 0 and the other cluster to 1 intensity units, scanning each A-line and marking the first index, which is 1 intensity unit as the start of the lumen outline,

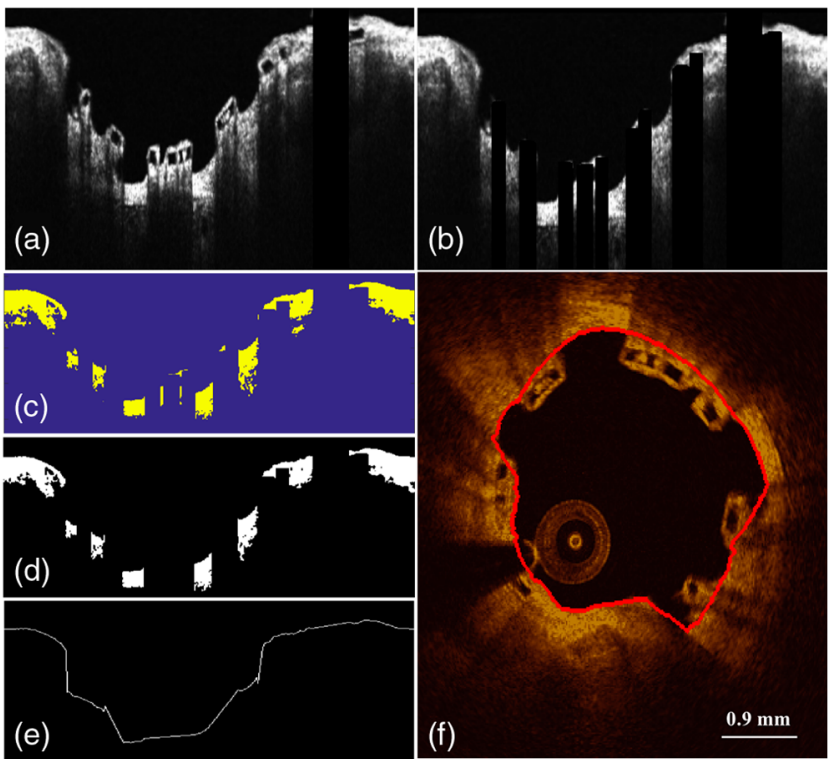

Fig. 3 Automated detection of the lumen border applied to an image with BVS struts: (a) OCT image cleared of imaging artifacts, (b) image cleared of the detected struts, (c) $K$-means segmentation masked image (with $k=2$ clusters), (d) binary thresholded image, (e) lumen border outline detected using linear interpolation, and (f) lumen border translated into Cartesian coordinates in red.

and connecting the points using a linear interpolation in polar coordinates, yielding a curve in Cartesian coordinates.

\section{Dataset}

For the validation of the proposed plaque characterization method, we used anonymized OCT examinations from 15 patients (Hospital Universitario Marqués de Valdecilla, Santander, Spain). These patients ranged from a day postprocedure to almost 9 months postprocedure. The purpose of using this longitudinal range was to include OCT images with a combination of malapposed, well-apposed, and embedded struts. The images were acquired using frequency domain (FD-OCT) OCT equipment (LightLab Imaging, Inc.) with a 6 Fr FD-OCT catheter (C7 Dragonfly). Automated contrast injection was performed to optimize the best image quality in all pullbacks. The endovascular polymeric implant used for this study is "The Absorb Vascular Scaffold BVS by Abbott Vascular, Santa Clara, California, USA," a $150 \mu \mathrm{m}$ think bioresorbable poly-L-lactic acid scaffold with a $7 \mu$ m thick bioresorbable poly (D, L-lactide) coating, which elutes everolimus. The scaffold used has a diameter between 2.5 and $3.5 \mathrm{~mm}$ and is 12 to $28 \mathrm{~mm}$ in length. An expert manually marked the BVS strut outer border and the lumen border outlines on 1140 images, which were used as the gold standard for the present study. These images were marked to count the number of BVS struts visible. Two medical experts annotated the same images, and there was disagreement for only five images, which were discarded from the study. Given the lack of interobserver variability, we used annotations from one of the experts who marked the outer border points of each strut to annotate its position. Struts with multiple bright spots inside the strut were still considered as one strut. Then, we connected the marked points and fit them to a circle and ellipse whose area was the gold standard device coverage area used in the validation. Approximately $5 \%$ of all frames were removed from the validation study for the following reasons: experts' disagreement (five frames), excess blood inside the 
Table 1 Summary of validation metrics for BVS strut detection.

\begin{tabular}{lc} 
Validation metric & Value across 1140 images \\
\hline Pearson correlation coefficient & 0.88 \\
PPV & 0.93 \\
TPR & 0.90 \\
FDR & 0.07 \\
FNR & 0.10 \\
F1 score & 0.91 \\
\hline
\end{tabular}

lumen and around struts due to improper flushing, presence of bifurcations obscuring the luminal outline, and presence of multiple scaffolds on top of one another. The data were obtained under ethical procedures and screened for patient consent.

\section{Results}

To validate our proposed methodology, we used common validation metrics (Table 1) for the strut count and performed a Bayesian regression and Bland-Altman analysis for the strut count and area and the lumen area.

\subsection{Bayesian Parameter Estimation}

In this work, we determine the optimal regression parameters $(m, c)$ using Bayesian methods. We calculate the probability distribution of each regression parameter given the observed results; then using Bayes theorem, we can denote

$P(m, c, \sigma \mid D)=\frac{P(D \mid m, c, \sigma) \cdot P(m, c, \sigma)}{P(D)}$,

where $\mathrm{m}$ and $\mathrm{c}$ are the regression gradient and $y$-intercept, respectively, $\sigma$ is the standard deviation for the Gaussian likelihood, D is the data and consists of the area of strut count detected by the methodology and annotated by the expert, $P(D \mid m, c, \sigma)$ is the likelihood of getting the observed data given the parameters, $P(m, c, \sigma)$ is the prior belief on the values of the parameters, and $P(D)$ is the normalization constant. We expect the detected area or strut count for the $i$ 'th image to be linearly proportional to that found by annotations, namely $\mathrm{D}_{\mathrm{i}}=\mathrm{A}_{\mathrm{i}} \mathrm{m}+\mathrm{c}$ for some parameters $m$ and $c$. We assume a Gaussian likelihood with some variance $\sigma^{2}$; then, our likelihood function is given by

$P(D \mid m, c, \sigma)=\frac{1}{\left(2 \pi \sigma^{2}\right)^{\frac{n}{2}}} \prod_{i=1}^{n} \exp \left[-\frac{1}{2 \sigma^{2}}\left(D_{i}-A_{i}\right)^{2}\right]$,

where $n$ is the number of images, $D_{i}$ is the strut count or area detected by the methodology, and $A_{i}$ is the strut count or area annotated by the expert. We assume a uniform prior distribution for the parameters $m$ and c and a Jeffrey's prior for the scale parameter $\sigma$, which yields the prior probability to be

$P(m, c, \sigma) \propto \frac{1}{\sigma}$.

Combining the above expressions, we use Markov Chain Monte Carlo (MCMC) to sample from the posterior distribution $P(m, c, \sigma \mid D)$ to find the optimal parameter values.
The purpose of this Bayesian analysis is to quantify the level of uncertainty in the regression parameters to holistically evaluate the accuracy of the proposed methodology. The regression $y$-intercept corresponds to the systematic error in the proposed methodology and the gradient corresponds to the ratio of the detected strut count or area to the gold standard. We expect the $y$-intercept and gradient to be 0 and 1, respectively, for a perfect detection. Using the posterior distribution allows quantification of how much these parameters deviate from this null hypothesis.

\subsection{BVS Strut Count}

To validate the proposed BVS detection methodology, we used common validation metrics (Table 1). We denote the true positives (TP) as the struts detected by both the methodology and expert, FPs as those struts detected by the methodology but not by the expert, and false negatives (FNs) as the struts detected by the expert but missed by our methodology. Using these definitions, we calculated the validation metrics in Table 1 . The metrics in Table 1 are defined as follows: the PPV is defined as TP/ $(\mathrm{TP}+\mathrm{FP})$, the true positive rate (TPR) is defined as TP/(TP + $\mathrm{FN}$ ), the false discovery rate (FDR) is defined as $1-\mathrm{PPV}$, the false negative rate (FNR) is defined as $1-\mathrm{TPR}$, and the F1 score is defined as $2 \mathrm{TP} /(2 \mathrm{TP}+\mathrm{FP}+\mathrm{FN})$.

Additionally, we performed a Bayesian regression [Figs. 4(a)-4(c)] and Bland-Altman analysis [Fig. 4(d)] for the strut count. The Bland-Altman analysis revealed a mean of -0.16 , which shows no significant bias. From the regression posterior distributions [Figs. 4(b) and 4(c)], the systematic error is a sharply peaked Gaussian at 0.5 , which is a negligible systematic error and the gradient is a sharply peaked Gaussian at 0.91 , depicting that up to $91 \%$ of the struts are detected by our methodology compared with the gold standard. We also calculated the validation metrics and error in strut detection for each patient as in Fig. 5. We see that all patients except patient 9 have a narrow distribution centered at approximately 0 with a small tail [Fig. 5(a)], indicating a low detection error.

\subsection{BVS Strut Area Measure}

Additionally, we also calculated the area of the circle and ellipse that best fit the outer borders of the struts. To find the best-fit circle, we used Pratt's method on images with at least three detected struts spaced sufficiently apart to generate an arc to approximate a circle or five points to approximate an ellipse. To find the best-fit ellipse, we used a nonlinear least-squares criterion using the conic representation of an ellipse given by

$a_{1} x^{2}+a_{2} y^{2}+a_{3} x+a_{4} x y+a_{5} y=0$.

The regression analysis [Fig. 6(a)] revealed an $R^{2}$ value of 0.93. The Bland-Altman analysis [Fig. 6(b)] revealed a mean of $0.14 \mathrm{~mm}^{2}$ for the scaffold area, which shows no significant bias. From the regression posterior distributions [Figs. 6(c) and $6(\mathrm{~d})$ ], the systematic error is Gaussian centered at $0.15 \mathrm{~mm}^{2}$ and the relative accuracy is $\sim 95 \%$.

Quantifying the ratio of the annotated strut area to the detected strut area for each patient, the violin plot captures the distribution for each patient and shows that the majority of patients have a mean centered close to 1 with a small tail [Fig. 7(a)]. Analyzing the distribution of $S A_{A}-S A_{M}$ revealed a sharply peaked Gaussian centered at $0.14 \mathrm{~mm}^{2}$, indicating 

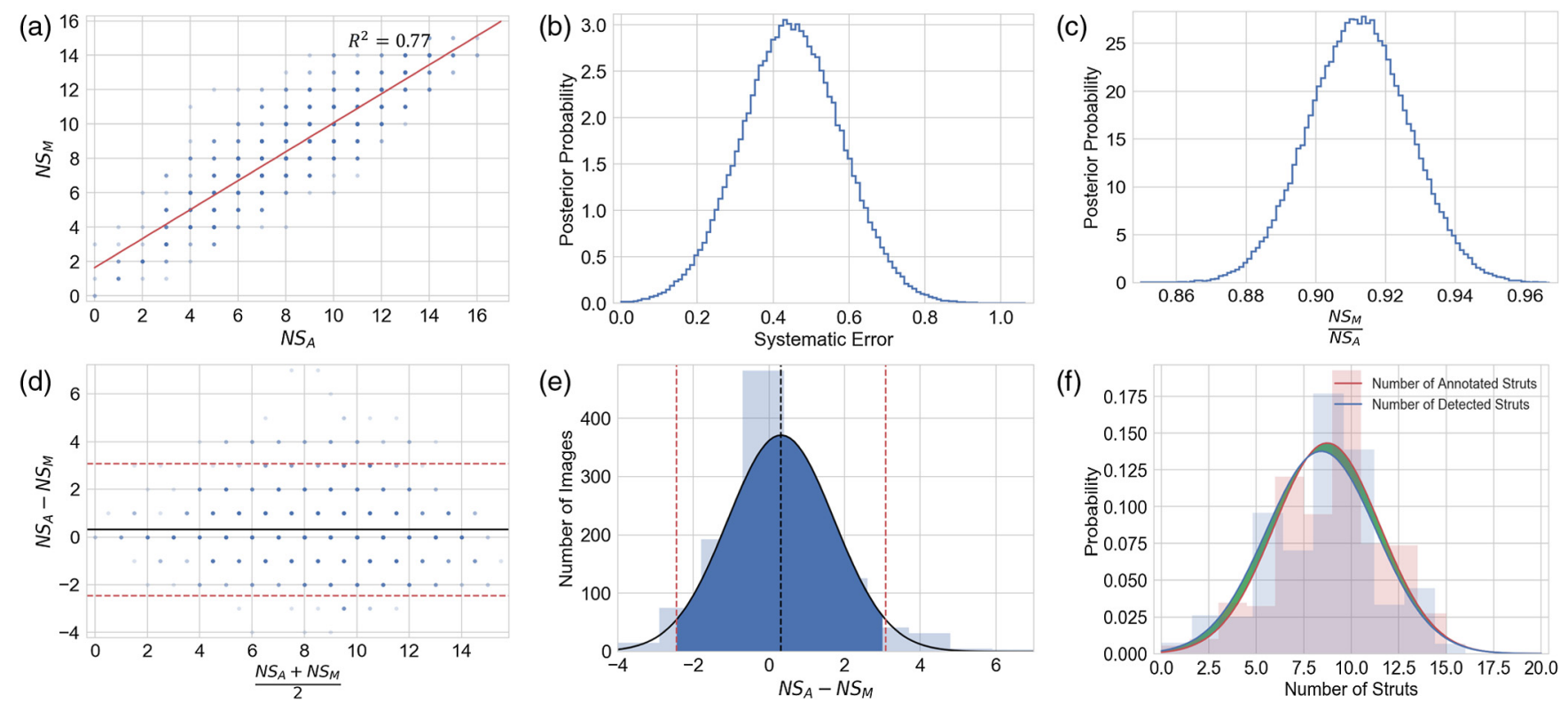

Fig. 4 Summary of overall results for BVS strut detection using binary validation. (a) Regression plot of the number of struts detected by the methodology versus those identified by the expert, where the color density of each point corresponds to the number of points accumulated at that coordinate. (b) Posterior distribution of the $y$-intercept from the regression analysis, (c) posterior distribution of the gradient of the regression analysis, (d) Bland-Altman analysis with the mean (solid black line) and the $95 \%$ confidence interval (dashed red lines), (e) histogram with a Gaussian best fit for the difference in the number of struts detected by methodology and those detected by the expert annotations, with the mean (dashed black line) and the $95 \%$ confidence interval (dashed red lines), and (f) histogram with a Gaussian fit for the number of struts annotated by experts (red) and those detected by our methodology (blue); the shaded green region is the difference between the two $\left(\mathrm{NS}_{\mathrm{A}}\right.$ : number of struts annotated by experts, $\mathrm{NS}_{\mathrm{M}}$ : number of struts detected by our methodology). The posterior probability in (b) and (c) is not normalized and is dimensionless. The probability in (f) is normalized and is between 0 and 1.
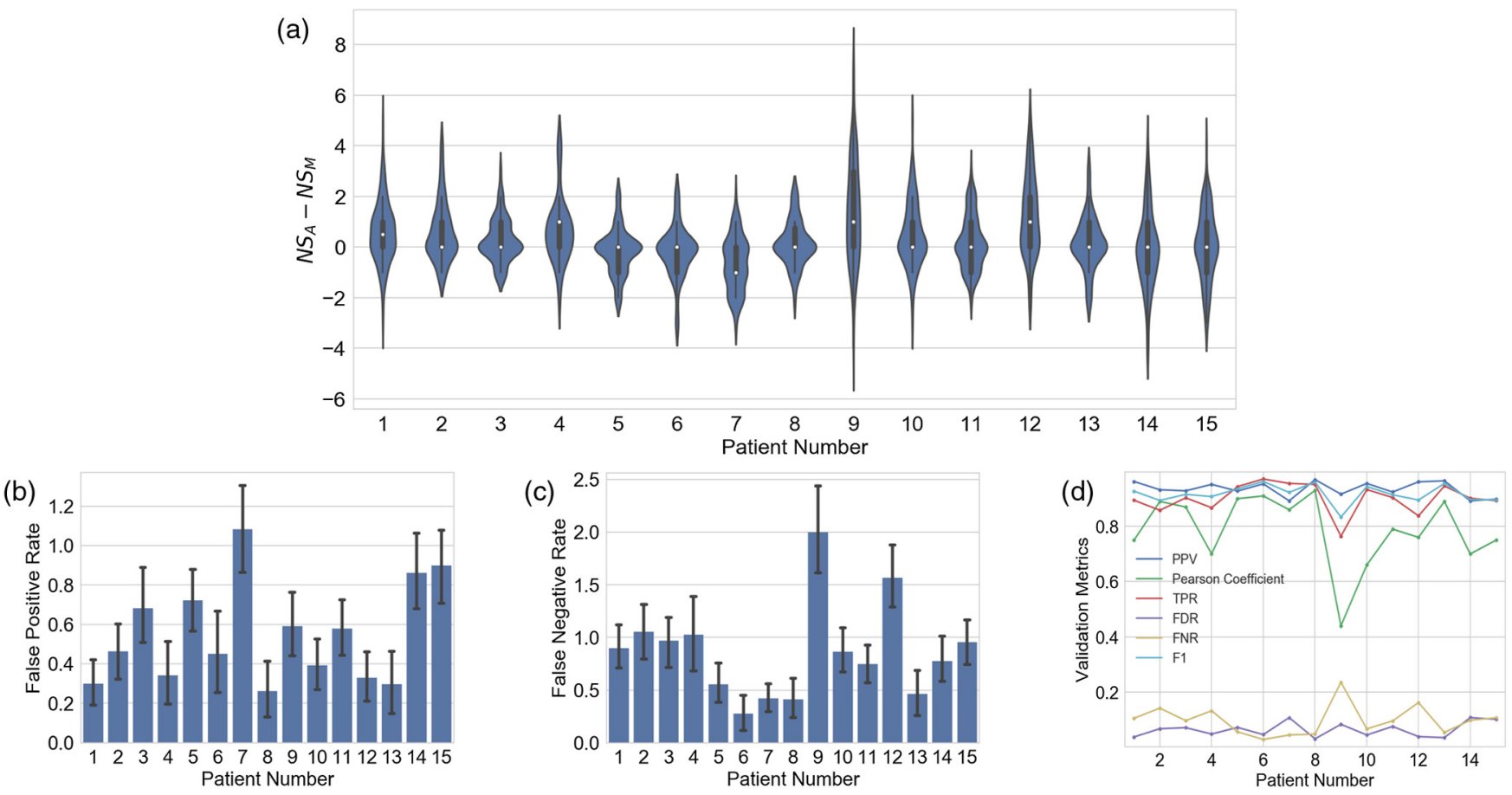

Fig. 5 Summary of error and validation metrics for BVS strut detection using binary validation for each patient. (a) A violin-plot outlining the error in strut detection for each patient; the white dot indicates the mean, the black tube surrounding this dot represents the $95 \%$ confidence interval, and the shape of each violin shows the distribution, (b) FPR, (c) FNR for each patient, and (d) summary of validation metrics (PPV, Pearson correlation coefficient, TPR, FDR, FNR, and the F1 score) from Table 1 for all patients $\left(\mathrm{NS}_{\mathrm{A}}\right.$ : number of struts annotated by experts, $\mathrm{NS}_{\mathrm{M}}$ : number of struts detected by our methodology). 
(a)

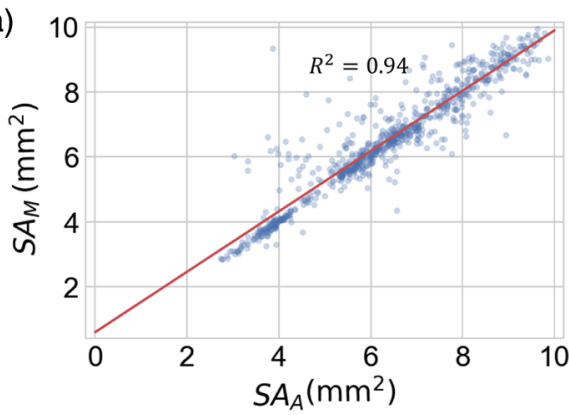

(c)

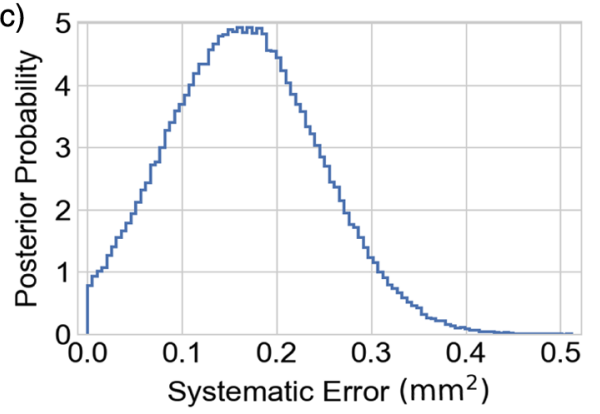

(b)

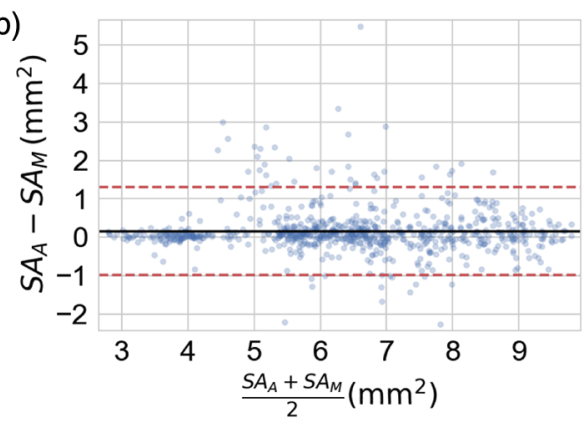

(d)

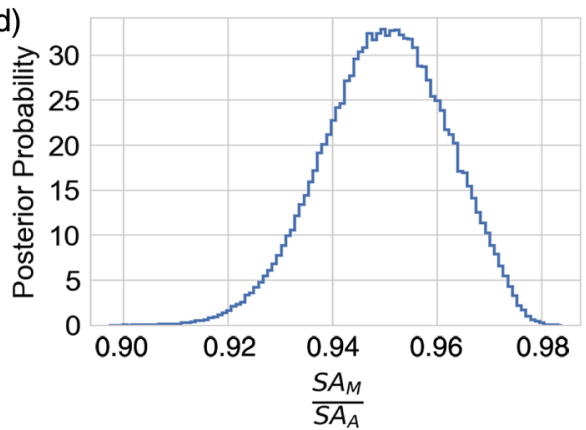

Fig. 6 Summary of BVS strut area validation. (a) Regression plot of the average area of the best fit ellipse and circle for the BVS struts outer border as detected by our methodology versus that annotated by an expert, (b) Bland-Altman analysis with the mean (solid black line) and the $95 \%$ confidence interval (dashed red lines), (c) posterior distribution of the $y$-intercept from the regression analysis, and (d) posterior distribution of the gradient of the regression analysis $\left(\mathrm{SA}_{\mathrm{A}}\right.$ : the average area of the best fit circle and ellipse through the annotated strut outer borders, $\mathrm{SA}_{\mathrm{M}}$ : the average area of the best fit circle and ellipse through the detected strut outer borders). The posterior probability in (c) and (d) is dimensionless and is not normalized.

a reliable detection result [Fig. 7(b)]. We define the detection area error for each patient as follows:

$\frac{\left|A_{A}-A_{M}\right|}{A_{A}} \times 100 \%$,

where $A_{A}$ is the annotated lumen/BVS strut area and $A_{M}$ is the detected lumen/BVS strut area. We calculated the detection error for each patient using Eq. (9) to quantify the normalized error with respect to the true area [Fig. 7(c)] and found that most patients had a detection error of less than $5 \%$ on average. Furthermore, we found that the ratio of the annotated to detected strut area is a sharply peaked curve around 1 for almost all patients [Fig. 7(a)], indicating a low area error in strut detection.

\subsection{Lumen Detection}

To validate the lumen detection component of our methodology, we compared the area of the detected versus annotated lumen outline. The regression analysis [Fig. 8(a)] revealed a $R^{2}$ of 0.94 and a mean of $0.09 \mathrm{~mm}^{2}$ for the lumen area, indicating no significant bias. Because the lumen detection relies on previous processing stages, this bias can be attested to errors propagated from previous steps. This limitation is further discussed in Sec. 4.6. From the regression posterior distributions [Figs. 8(c) and 8(d)], the systematic error is a sharply peaked Gaussian around $0.2 \mathrm{~mm}^{2}$ and the relative accuracy is $\sim 95 \%$.

The ratio of the annotated to the detected lumen area for each patient shows that the majority of patients have a mean centered at $\sim$ 1. with a small tail [Fig. 9(a)]. Analyzing the distribution of $\mathrm{LA}_{\mathrm{A}}-\mathrm{LA}_{\mathrm{M}}$ revealed a sharply peaked Gaussian centered at
$0.09 \mathrm{~mm}^{2}$. We also calculated the lumen area detection error for each patient [Eq. (9)] to quantify the normalized error with respect to the true area and found that most patients had a detection error of less than $10 \%$ on average [Fig. 9(c)].

\subsection{Application Examples and Comparison with the Literature}

We applied our algorithm to 1140 images on an i7 8G RAM machine; detection time scales linearly with the number of OCT images and took $3 \mathrm{~s}$ per OCT image. This amounts to $\sim 5$ min per patient (with 100 frames). In Figs. 10(a)-10(c), we show our algorithm applied to images with embedded, malapposed, and well-apposed struts.

Existing strut detection methods cannot be used to detect struts in patients with a combination of embedded and apposed struts; therefore, we cannot quantitatively compare our strut detection algorithm to literature methods. To be able to have a direct comparison of the lumen detection part of our method with existing methods, we implemented and applied on our dataset the most recent and robust method presented in the literature. ${ }^{25}$ We performed a regression analysis [Fig. 11(a)] and found that our algorithm has an $R^{2}=0.94$, while the literature method has an $R^{2}=0.86$. From the Bland-Altman analysis [Fig. 11(b)], we found that our algorithm shows no significant bias $\left(0.09 \mathrm{~mm}^{2}\right)$ while the literature algorithm had a significant bias $\left(0.44 \mathrm{~mm}^{2}\right)$. The Bland-Altman analysis also revealed that the literature algorithm has a significantly larger confidence interval band indicating low detection reliability. We also compared the lumen area detection error per patient [Fig. 11(c)] and 


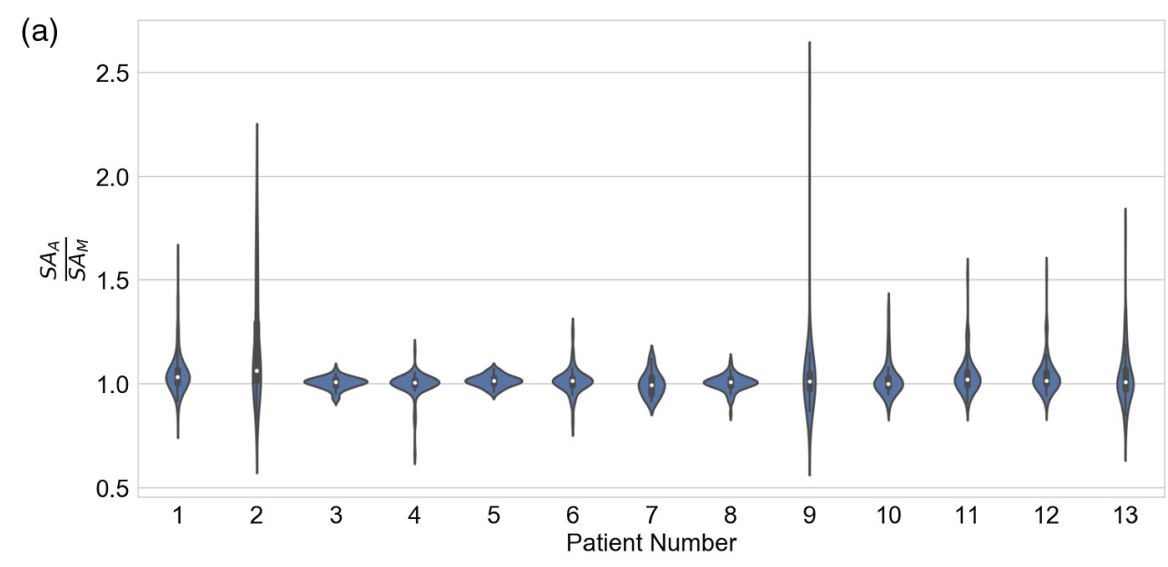

(b)

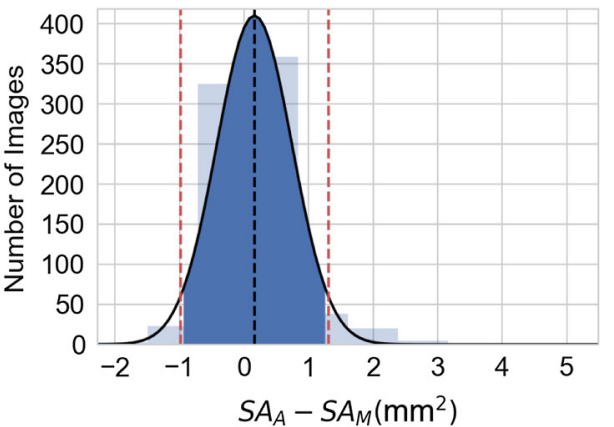

(c)

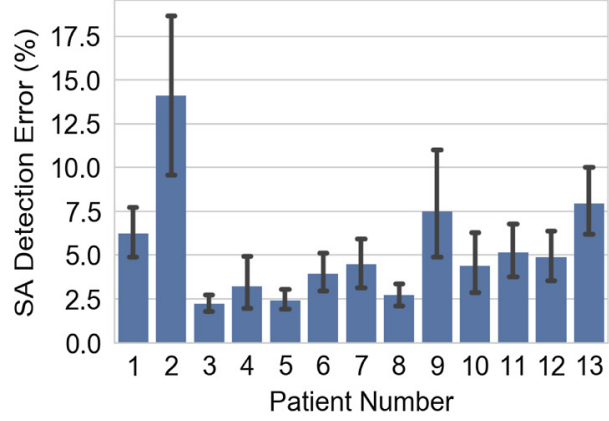

Fig. 7 Summary of BVS strut area detection error. (a) A violin-plot outlining the error in strut area detection for each patient; the white dot indicates the mean, the black tube surrounding this dot represents the $95 \%$ confidence interval, and the shape of each violin shows the distribution, (b) histogram with a Gaussian fit for the difference in scaffold detection area between the annotations and methodology, and (c) BVS strut area detection error Eq. (9) for each patient $\left(\mathrm{SA}_{\mathrm{A}}\right.$ : the average area of the best fit circle and ellipse through the annotated strut outer borders, $\mathrm{SA}_{\mathrm{M}}$ : the average area of the best fit circle and ellipse through the detected strut outer borders).

(a)

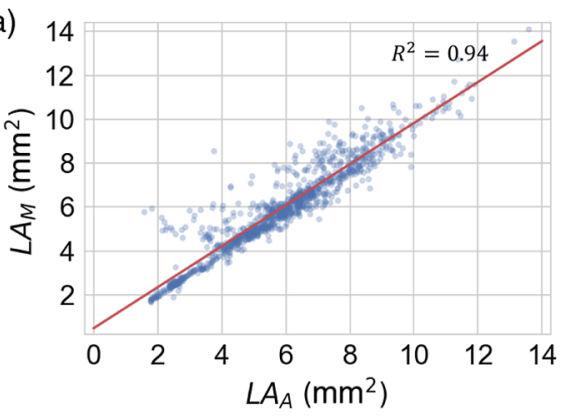

(c)

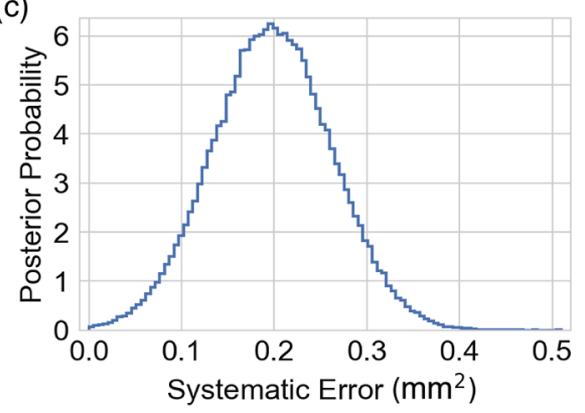

(b)

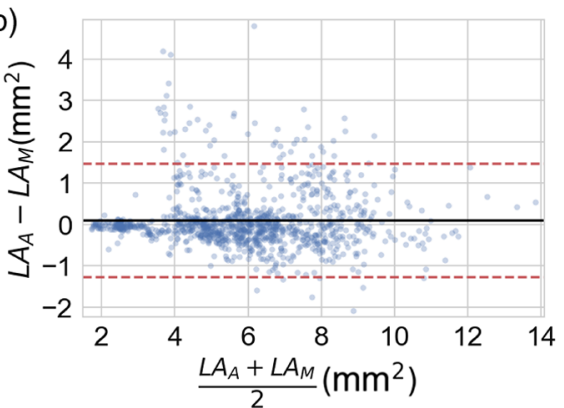

(d)

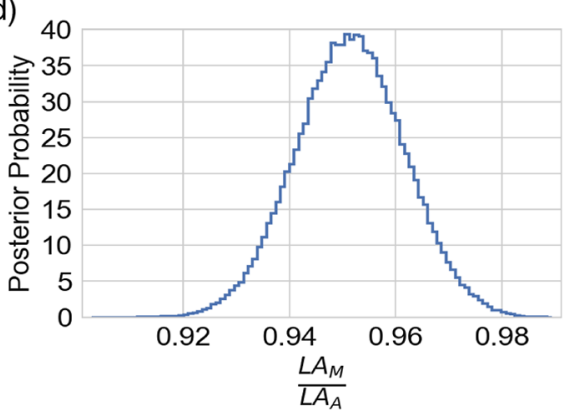

Fig. 8 Summary of lumen detected area validation. (a) Regression plot of the lumen area for the methodology versus annotations, (b) Bland-Altman analysis with the mean (solid black line) and the 95\% confidence interval (dashed red lines), (c) posterior distribution of the $y$-intercept from the regression analysis, and $(\mathrm{d})$ posterior distribution of the gradient of the regression analysis $\left(\mathrm{LA}_{\mathrm{A}}\right.$ : the area of the lumen annotated by experts, $\mathrm{SA}_{\mathrm{M}}$ : the area of the lumen detected by the methodology). The posterior probability in (c) and (d) is dimensionless and is not normalized. 
(a)

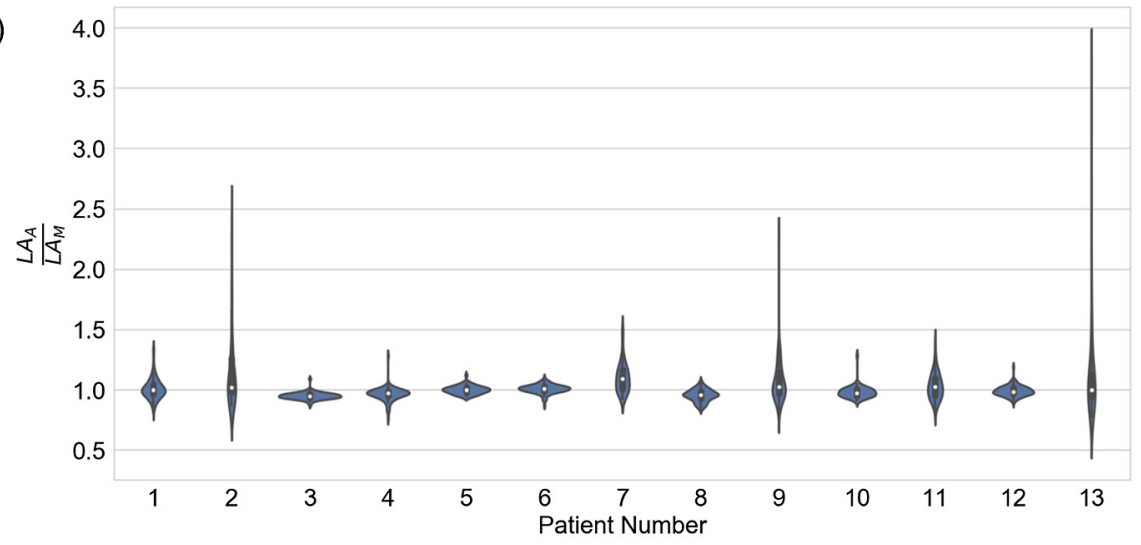

(b)

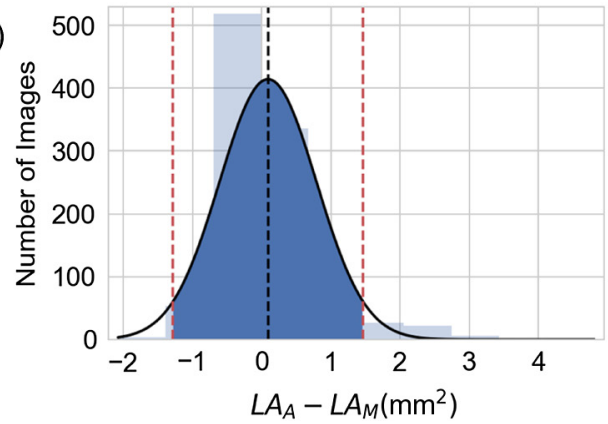

(c)

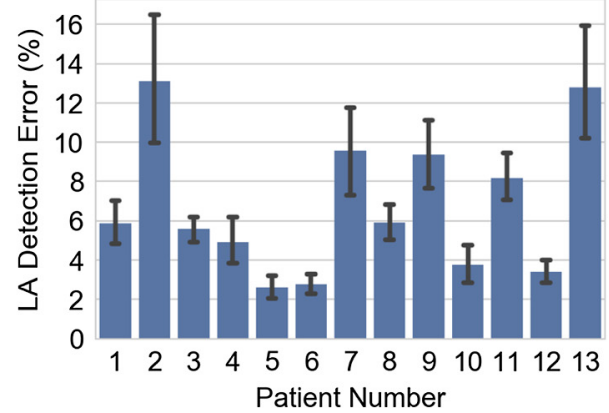

Fig. 9 Summary of lumen area detection error. (a) A violin-plot outlining the error in lumen area detection for each patient; the white dot indicates the mean, the black tube surrounding this dot represents the $95 \%$ confidence interval, and the shape of each violin shows the distribution, (b) histogram with a Gaussian fit for the difference in lumen detection area between the annotations and methodology, and (c) lumen area detection error [Eq. (9)] for each patient $\left(L A_{A}\right.$ : area of the annotated lumen, $L A_{M}$ : the area of the lumen detected by the methodology).

found that the literature method produces significantly larger errors in most cases.

To study the applicability of the proposed methods when used by 3-D reconstruction algorithms, we analyzed the fraction of all frames that cannot be used by these algorithms. We define the frame failure rate (FFR) as the fraction of all segmented frames that cannot be used for further analysis (Fig. 12). In Fig. 12, the $x$-axis is the BVS strut/lumen area error and the corresponding fraction of all frames that cannot be used for $3-\mathrm{D}$ reconstruction. We fit the curves to a decaying exponential and found a decay rate of 0.22 and 0.15 for the strut and lumen detection FFR, respectively. Therefore, the FFR falls exponentially, indicating that most frames have a low detection error. The FFR drops more steeply for the strut detection [Fig. 12(b)] as compared with that for the lumen detection [Fig. 12(d)]. This difference indicates that there is a larger error in the lumen detection procedure, which can be attested to postprocessing error accumulation. The lumen detection algorithm uses the input from previous steps in the image processing pipeline (artifact removal and strut detection); consequently, any errors incurred in these earlier steps will be accumulated in the lumen detection method. ${ }^{34}$ Finally, we repeated the FFR analysis for the literature lumen detection method [Fig. 11(d)] and found that the FFR drops less slowly for the literature method ${ }^{25}$ as compared with our proposed algorithm. Together, these metrics show that our algorithm provides a greater and more reliable lumen detection accuracy, and our FFR analysis, validation plots, and representative examples together show the applicability of our proposed method. Given the high PPV of the strut
(0.93) and lumen detection (0.94) methodology, our proposed algorithm in conjunction with manual calibration holds promise of producing realistic 3-D geometric models of the scaffolded coronary artery. ${ }^{35}$

We also applied our algorithm to images with excess noise and blood artifacts in the lumen and found accurate detection results [Figs. 13(a) and 13(b)]. In some images, the orientation of the struts precludes light from interacting with the strut edge, and, as a result, the struts are seen as incomplete due to this mismatch in the refraction index-we call these struts incomplete. We tested our algorithm on images with incomplete struts and found that it accurately detects them [Fig. 13(c)].

\subsection{Limitations}

Our algorithm produces FPs when there is a shadow behind the strut and the back face of the strut cannot be seen properly and in images where there is a bright fringe inside the strut. In both cases, single struts are identified as two distinct struts as the algorithm interprets the fringe as dividing the strut. The algorithm produces FNs in the presence of bright edges, dark shadows, or with struts that are resorbed or fractured. A $K$-means clustering algorithm differentiates between strut images with bright or dark edges, and, through judicious management of cluster number, we can work to optimize PPV of the algorithm. Images with the vascular scaffold and a metallic stent implant in the same frame can often induce black shadows, especially behind the metallic struts and at the edge of the BVS. Consequently, BVS struts, which are close to one another, may appear as a single strut, but this does not change the lumen detection. 

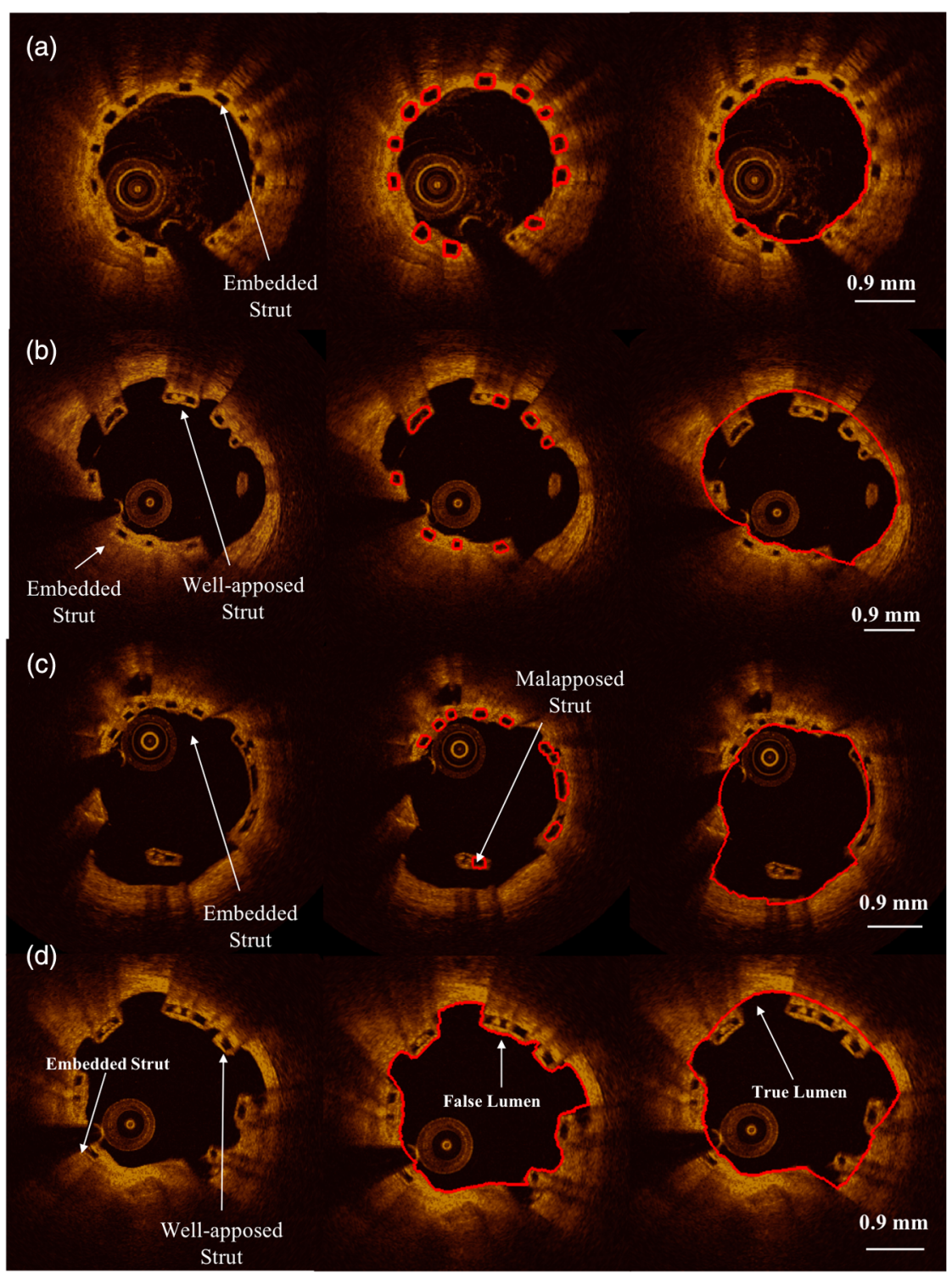

Fig. 10 Representative cases of our algorithm applied to images. (a) Example of algorithm applied to embedded struts, (b) example of algorithm applied to a combination of embedded and well-apposed struts, and (c) algorithm applied to images with embedded and malapposed struts. The first column is the raw OCT image, the second column is the detected struts, and the third column is the detected lumen boundary. (d) Example of lumen detection method using the proposed and traditional methods. ${ }^{25}$ The first image in (d) is a raw OCT image, the second it the lumen detected using a literature method, and the third is the lumen detected using our method.

Finally, our algorithm is sensitive to cases in which the imaging catheter pushes against the lumen border. The error in the detected lumen is a result of errors propagated from preprocessing steps. ${ }^{34}$ In these images, any error in the detection of the protective sheath propagates as an error in the lumen detection.

\section{Discussion}

We present an automated method to detect endovascular polymeric struts and the lumen border in intracoronary OCT images. Our proposed method accurately removes imaging artifacts via edge detection, ${ }^{16}$ utilizes a $K$-means clustering algorithm to automatically segment the image, identifies the polygonalshaped polymeric strut outlines, and accurately locates the true luminal border. We applied our methodology to BVS struts, tested our method on 1140 images, and found a PPV of 0.93 for strut detection. Additionally, we found an $R^{2}$ correlation of 0.94 between the detected and annotated lumen area. We also performed a Bayesian analysis using MCMC on the regression parameters to quantify the level of uncertainty in the validation. This yielded sharply peaked Gaussian posteriors, without longtails, which indicates that the parameter values are well defined and can be reliably used to assess the efficacy of presented methodology. Given the low validation errors, our methodology can be adapted to future endovascular polymeric implants, where the struts have a polygonal core and well-defined edges.

Existing polymeric strut detection methods use different thresholds to detect polymeric struts above and below the arterial wall, ${ }^{16}$ which leads to problems in follow-up patients 
(a)

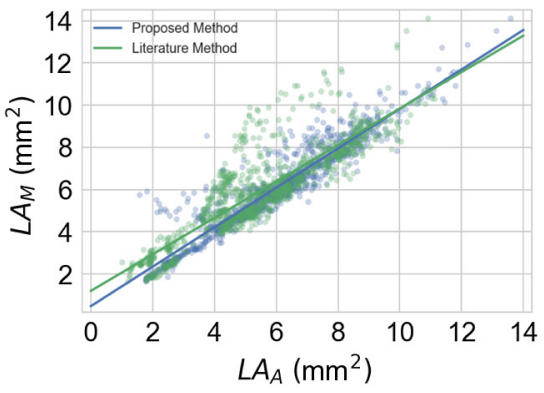

(c)

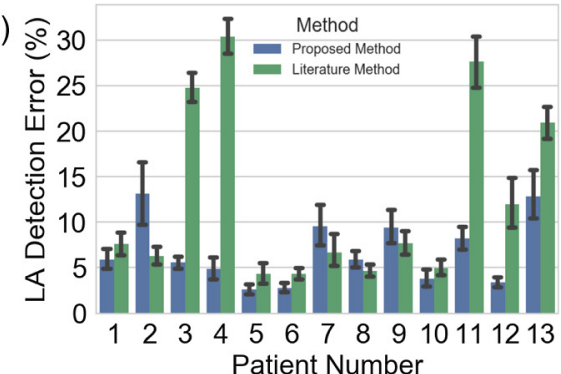

(b)

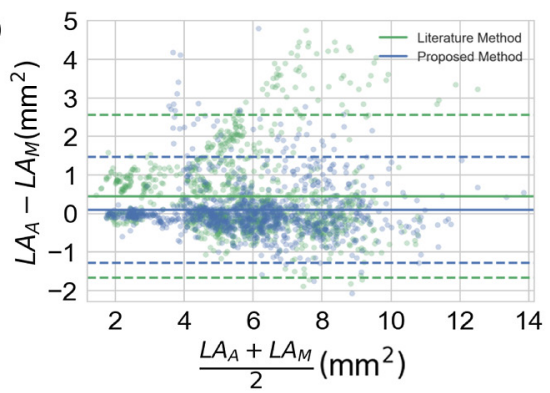

(d)

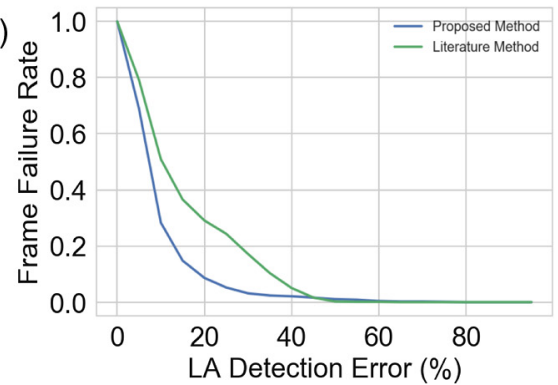

Fig. 11 Validation plots to compare proposed lumen detection algorithm to the literature lumen detection algorithm. ${ }^{25}$ (a) Regression plot for the lumen area detected by the methodology (literature ${ }^{25}$ and proposed lumen detection algorithm) versus the lumen area annotated by expert observations, (b) BlandAltman analysis for literature ${ }^{25}$ and proposed algorithm with the solid line indicating the mean and the dashed lines indicating the 95\% confidence interval, (c) lumen area detection error [Eq. (9)] for each patient for literature ${ }^{25}$ and proposed algorithm, and (d) the average FFR across all images versus the lumen detection area error for literature ${ }^{25}$ and proposed algorithm $\left(\mathrm{LA}_{\mathrm{A}}\right.$ : area of the annotated lumen, $L A_{M}$ : the area of the lumen detected by the methodology). The FFR is dimensionless.

who have a combination of struts above and below the arterial wall. Furthermore, existing lumen detection algorithms ${ }^{25}$ fail to accurately detect the true luminal outline in images with the apposed vascular scaffolds [Fig. 10(d)]. To our knowledge, there

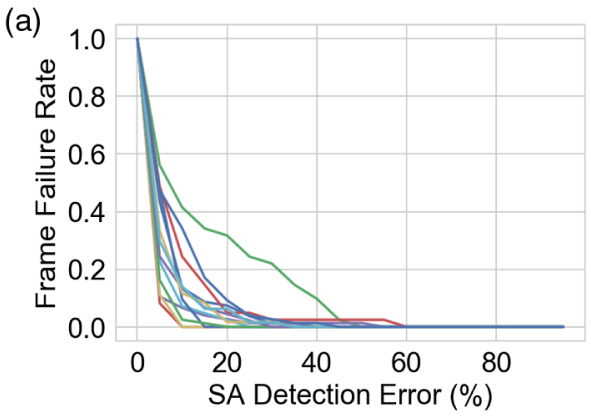

(c)

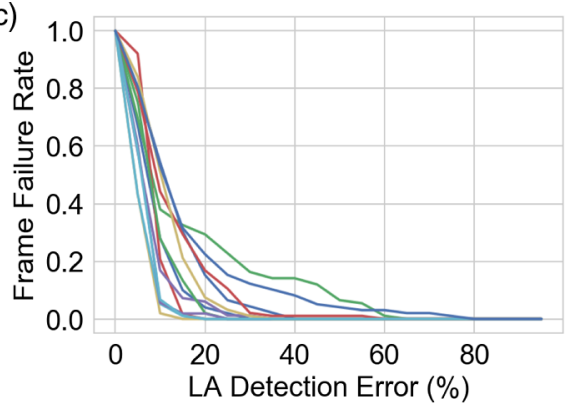

is no fully automated method to accurately detect the lumen in scaffolded OCT images. We propose an automated procedure to detect both BVS struts independent of strut position and the lumen border in images with an apposed vascular scaffold. Our

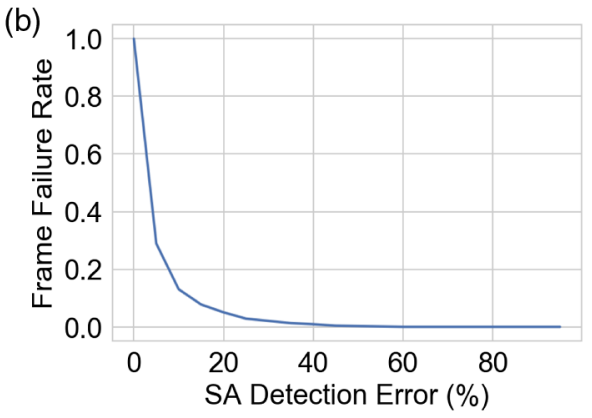

(d)

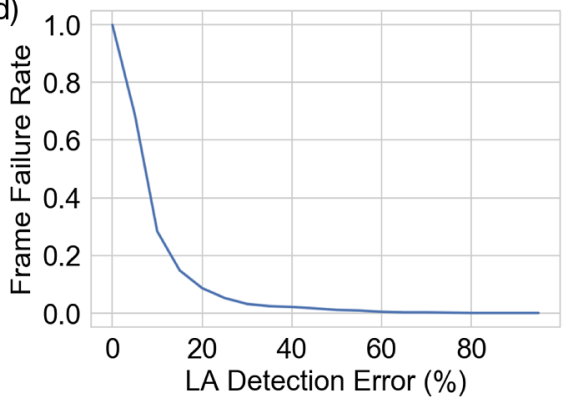

Fig. 12 Summary of the FFR for the polymeric strut and lumen detection methodology. (a) The FFR versus the BVS strut detection area error for each patient, (b) the average FFR across all images versus the BVS strut detection area error, (c) the FFR versus the lumen detection area error for each patient, and (d) the average FFR across all images versus the lumen detection area error. In panels (a) and (c), the different colors represent each of the 13 patients (SA: average area of the best fit circle and ellipse through detected BVS strut outer borders, LA: lumen area). The FFR is dimensionless. 


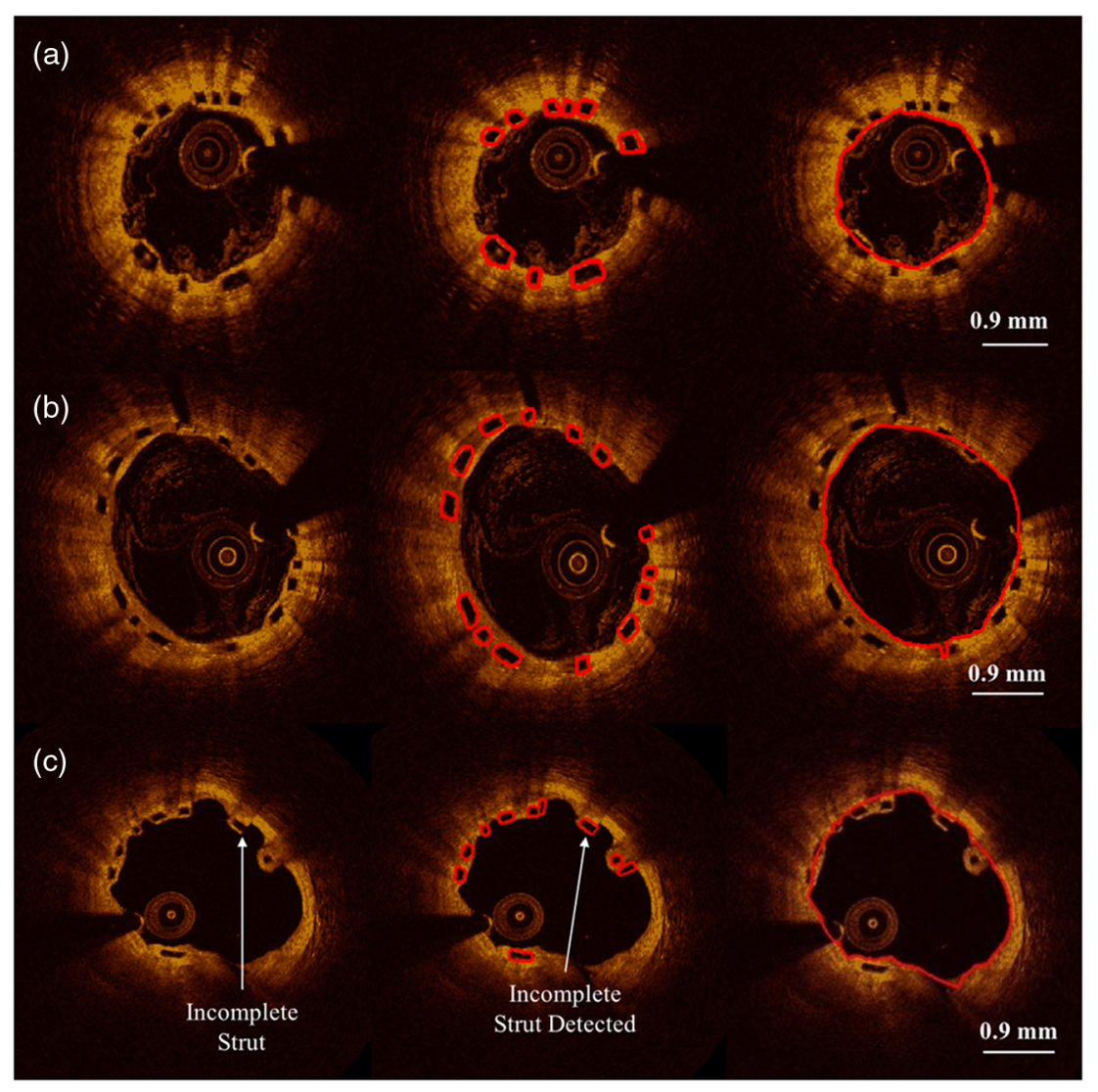

Fig. $13(a, b)$ Example of algorithm applied to cases with blood artifacts, noise, poor image quality, and (c) incomplete struts. The first column of each image is the raw OCT image, the second column shows the detected struts, and the third column shows the detected lumen border.

approach is crucial because analysis of acute vascular response to polymeric implants requires a combination of both struts above and below the arterial wall. ${ }^{36,37}$ Our procedure scales linearly in the number of OCT images and requires less than 3 s per OCT image on an i7 8 GB RAM machine, which requires $\sim 5 \mathrm{~min}$ (for 100 scaffolded frames) per patient and eliminates the need for clinicians to laboriously segment the struts by hand.

The spatial location of the polymeric struts is of interest to clinicians because it can be used to calculate the diameter of the implants and position of the struts with respect to that of the lumen together these metrics can be used to quantify the overall coverage of the device and procedural success. This spatial analysis can provide relevant quantitative metrics to identify specific struts with higher risk of thrombosis allowing for timely clinical interventions. ${ }^{38}$ Registering spatial positioning of struts is the cornerstone to studying how polymeric struts evolve over time. Such temporal tracking is instrumental to assessing the efficacy of the emerging polymeric implant technology as it can be combined with other techniques, such as plaque characterization $^{29}$ to study hemodynamic evolution over time. ${ }^{39}$

The proposed polymeric strut and lumen detection methodology is also pivotal to researchers interested in studying the biomechanics and rheology inside a mechanically scaffold-supported coronary artery. To accurately describe the biomechanical environment of a coronary artery, a 3-D geometry of the device struts and lumen outline is necessary. ${ }^{40}$ By applying the proposed method to BVS, one can accurately detect the outline of BVS struts and the luminal border in each OCT frame, and this result can be translated to produce a 3-D geometry using well-known 3-D OCT reconstruction algorithms. ${ }^{41}$ To assess the feasibility of this translation, we performed a frame failure analysis, which uses a series of different threshold error rates and calculates the fraction of segmented frames that need to be discarded. We found that the FFR drops exponentially [Figs. 12(a)-12(d)], and this steep drop indicates a low detection error. Given this FFR error analysis in addition to other validation metrics (PPV, regression, and Bland-Altman analysis), our methodology with minimal manual calibration to remove erroneous frames can be translated to produce a 3-D geometry of the mechanically supported coronary artery.

Developing a patient-specific 3-D model will allow researchers to run structural and fluid dynamics computations within the stented coronary artery. ${ }^{42}$ This crucial analysis will provide clinicians with quantitative metrics to assess cardiovascular function postendovascular polymeric scaffold implantation and isolate contributing biomechanical factors. While the current generation of bioresorbable scaffolds has been withdrawn from the market, numerous companies are actively working to develop the next generation of polymeric endovascular implants. We note that such an algorithm will become even more important as it will enable us and others to study the biomechanical and hemodynamic effects of these devices. We define here an algorithm that can be extended well beyond the idea of bioresorbable scaffolds and especially to first-generation devices.

\section{Conclusion}

We present a fully automated method to segment polygonal shaped polymeric struts and the luminal border in intracoronary 
OCT images. The proposed methodology was applied to BVS struts and validated against expert annotation using a Bayesian regression, Bland-Altman analysis, and common validation metrics. Our method proved to be computationally efficient and produced accurate results across all tested metrics. Detection of struts and lumen border is crucial for studying the efficacy of endovascular devices by building patient-specific 3-D models and performing biomechanical simulations. ${ }^{35}$ The validation results of the proposed methodology show that it can be used as a foundation for creating such models to mechanistically study and understand the effect of polymeric scaffolds on cardiovascular function. A holistic insight into cardiovascular function will allow us to move toward a continuum of more reliable and effective patient-specific treatment strategies.

\section{Disclosures}

There are no conflicts of interest.

\section{Acknowledgments}

This project was supported in part by funds from the Caltech Franz and Anne Nierlich Summer Undergraduate Research Fellowship awarded to J.M.A., George \& Marie Vergottis Foundation Fellowship at Harvard Medical School awarded to L.A., and R01 support from the National Institutes of Health (GM 49039) awarded to F.R. and E.R.E.

\section{References}

1. F. Sanchis-Gomar et al., "Epidemiology of coronary heart disease and acute coronary syndrome," Ann. Transl. Med. 4(13), 256-256 (2016).

2. G. Sangiorgi et al., "Arterial calcification and not lumen stenosis is highly correlated with atherosclerotic plaque burden in humans: a histologic study of 723 coronary artery segments using nondecalcifying methodology," J. Am. Coll. Cardiol. 31(1), 126-133 (1998).

3. J. L. Fleg et al., "Detection of high-risk atherosclerotic plaque," JACC Cardiovasc. Imaging 5(9), 941-955 (2012).

4. K. Thygesen et al., "Universal definition of myocardial infarction," Circulation 116(22), 2634-2653 (2007).

5. S. Sorrentino et al., "Everolimus-eluting bioresorbable scaffolds versus everolimus-eluting metallic stents," J. Am. Coll. Cardiol. 69(25), 30553066 (2017).

6. R. A. Byrne et al., "Report of a European Society of CardiologyEuropean Association of Percutaneous Cardiovascular Interventions task force on the evaluation of coronary stents in Europe: executive summary," 36, 2608-2620 (2015).

7. M. P. Savage et al., "Stent placement compared with balloon angioplasty for obstructed coronary bypass grafts," N. Engl. J. Med. 337(11), 740-747 (1997).

8. P. W. Serruys et al., "Arterial remodeling after bioresorbable scaffolds and metallic stents," J. Am. Coll. Cardiol. 70(1), 60-74 (2017).

9. T. Tada et al., "Risk of stent thrombosis among bare-metal stents, firstgeneration drug-eluting stents, and second-generation drug-eluting stents," JACC Cardiovasc. Interv. 6(12), 1267-1274 (2013).

10. T. Okamura et al., "3-dimensional optical coherence tomography assessment of jailed side branches by bioresorbable vascular scaffolds: a proposal for classification," JACC Cardiovasc. Interv. 3(8), 836-844 (2010).

11. S. Cassese and A. Kastrati, "Bioresorbable vascular scaffold technology benefits from healthy skepticism," J. Am. Coll. Cardiol. 67(8), 932-935 (2016).

12. J. J. Wykrzykowska et al., "Bioresorbable scaffolds versus metallic stents in routine PCI," N. Engl. J. Med. 376(24), 2319-2328 (2017).

13. J. Bil and R. J. Gil, "Bioresorbable vascular scaffolds-what does the future bring?" J. Thorac. Dis. 8(8), E741-E745 (2016).

14. F. Alfonso et al., "Bioresorbable vascular scaffolds in patients with acute myocardial infarction: a new step forward to optimized reperfusion?" J. Thorac. Dis. 8(6), E417-E423 (2016).
15. D. Mukherjee, "Device thrombosis with bioresorbable scaffolds," N. Engl. J. Med. 376(24), 2388-2389 (2017).

16. A. Wang et al., "Automatic detection of bioresorbable vascular scaffold struts in intravascular optical coherence tomography pullback runs," Biomed. Opt. Express 5(10), 3589-3602 (2014).

17. J. G. Fujimoto et al., "Optical coherence tomography: an emerging technology for biomedical imaging and optical biopsy," Neoplasia 2(1-2), 9-25 (2000).

18. H. G. Bezerra et al., "Intracoronary optical coherence tomography: a comprehensive review," JACC Cardiovasc. Interv. 2(11), 1035-1046 (2009).

19. D. Stamper, N. J. Weissman, and M. Brezinski, "Plaque characterization with optical coherence tomography," J. Am. Coll. Cardiol. 47(8), C69C79 (2006).

20. A. M. Zysk et al., "Optical coherence tomography: a review of clinical development from bench to bedside," J. Biomed. Opt. 12(5), 051403 (2007).

21. H. S. Nam et al., "Automated detection of vessel lumen and stent struts in intravascular optical coherence tomography to evaluate stent apposition and neointimal coverage," Med. Phys. 43(4), 1662-1675 (2016).

22. K. Mandelias et al., "Automatic quantitative analysis of in-stent restenosis using FD-OCT in vivo intra-arterial imaging," Med. Phys. 40(6 Pt 1), 63101 (2013).

23. G. J. Ughi et al., "Automatic segmentation of in-vivo intra-coronary optical coherence tomography images to assess stent strut apposition and coverage," Int. J. Cardiovasc. Imaging 28(2), 229-241 (2012).

24. H. Lu et al., "Automatic stent detection in intravascular OCT images using bagged decision trees," Biomed. Opt. Express 3(11), 28092824 (2012).

25. L. Athanasiou et al., "Optimized computer-aided segmentation and 3D reconstruction using intracoronary optical coherence tomography," IEEE J. Biomed. Health Inf. PP, 1-1 (2017).

26. K. N. Chaudhury and S. D. Dabhade, "Fast and provably accurate bilateral filtering," IEEE Trans. Image Process. 25(6), 2519-2528 (2016).

27. C. Tomasi and R. Manduchi, "Bilateral filtering for gray and color images," in Sixth Int. Conf. on Computer Vision (IEEE Cat. No. 98CH36271), pp. 839-846, Narosa Publishing House (1998).

28. H. J. Blinchikoff and A. I. Zverev, Filtering in the Time and Frequency Domains, Noble Pub, Atlanta, Georgia (2001).

29. L. S. Athanasiou, D. I. Fotiadis, and L. K. Michalis, Atherosclerotic Plaque Characterization Methods Based on Coronary Imaging, Elsevier Science, London (2017).

30. E. W. Dijkstra, "A note on two problems in connexion with graphs," Numer. Math. 1(1), 269-271 (1959).

31. J. M. Amrute et al., "Automated segmentation of bioresorbable vascular scaffold struts in intracoronary optical coherence tomography images," in IEEE 17th Int. Conf. on Bioinformatics and Bioengineering, pp. 297302 (2017).

32. C. M. Bishop, Pattern Recognition and Machine Learning, Springer, New York (2006).

33. S. Kim et al., "Coronary stent fracture complicated multiple aneurysms confirmed by 3-dimensional reconstruction of intravascular-optical coherence tomography in a patient treated with open-cell designed drug-eluting stent," Circulation 129(3), e24-e27 (2014).

34. L. S. Athanasiou et al., "Error propagation in the characterization of atheromatic plaque types based on imaging," Comput. Methods Programs Biomed. 121(3), 161-174 (2015).

35. C. C. O'Brien et al., "Constraining OCT with knowledge of device design enables high accuracy hemodynamic assessment of endovascular implants," PLoS One 11(2), e0149178 (2016).

36. J. Gomez-Lara et al., "Serial analysis of the malapposed and uncovered struts of the new generation of everolimus-eluting bioresorbable scaffold with optical coherence tomography," JACC Cardiovasc. Interv. 4(9), 992-1001 (2011).

37. G. Giacchi et al., "Bioresorbable vascular scaffold implantation in acute coronary syndromes: clinical evidence, tips and tricks," Adv. Interv. Cardiol. 11, 161-169 (2015).

38. Y. Sotomi et al., "Quantitative assessment of the stent/scaffold strut embedment analysis by optical coherence tomography," Int. J. Cardiovasc. Imaging 32(6), 871-883 (2016).

39. A. I. Sakellarios et al., "Prediction of atherosclerotic plaque development in an in vivo coronary arterial segment based on a multilevel modeling approach," IEEE Trans. Biomed. Eng. 64(8), 1721-1730 (2017). 
40. I. Faik et al., "Time-dependent 3D simulations of the hemodynamics in a stented coronary artery," Biomed. Mater. 2(1), S28-S37 (2007).

41. L. S. Athanasiou et al., "3D reconstruction of coronary arteries using frequency domain optical coherence tomography images and biplane angiography," in Annual Int. Conf. of the IEEE Engineering in Medicine and Biology Society, pp. 2647-2650, IEEE (2012).

42. C. Chiastra et al., "Reconstruction of stented coronary arteries from optical coherence tomography images: feasibility, validation, and repeatability of a segmentation method," PLoS One 12(6), e0177495 (2017).

Junedh M. Amrute is an undergraduate at California Institute of Technology in his last year of his BS degree in bioengineering in the Division of Biology and Biological Engineering. He is interested in applying tools from engineering and physics to elucidate the mechanisms underlying cardiovascular disease. His research interests include physiological modeling, electrical impedance tomography to study atherosclerosis, noninvasive molecular imaging to detect hypoxia in tumor cells, and biomedical image processing.

Lambros S. Athanasiou received his diploma from the Department of Information and Communication Systems Engineering, University of the Aegean, Greece, in 2009 and his PhD from the Department of Materials Science and Engineering, University of Ioannina, Greece, in 2015 . He is currently working as a postdoctoral research fellow at the Institute for Medical Engineering and Science, MIT, Cambridge, Massachusetts. His research interests include medical image processing, biomedical engineering, decision support, and medical expert systems.

Farhad Rikhtegar received his master's degree at Sharif University of Technology in Tehran and his PhD in mechanical engineering from
ETH Zurich, where he conducted research on hemodynamics and drug transport in stented coronary arteries. He is currently a postdoctoral fellow in Harvard-MIT HST Division. He is interested in a mechanistic understanding of human pathophysiology, translation of preclinical experiments to clinical practices, design and optimization of medical devices, and developing translational engineering platforms.

Jose M. de la Torre Hernandez received his MD, PhD, and FESC and is an interventional cardiologist and head of the CV interventional department in the Hospital Universitario Marques de Valdecilla, in Santander, Spain. He is a member of the scientific committee of the Spanish Society of Cardiology and fellow of the European Society of Cardiology. $\mathrm{He}$ is an associate director of the Trans Catheter Therapeutics Meeting in the United States. His research interests include intravascular imaging, coronary artery disease, and drug eluting stents.

Tamara García Camarero is an interventional cardiologist working at Hospital Universitario Marqués de Valdecilla, in Santander, Spain. She is a member of the scientific committee of the Spanish Society of Cardiology and is a fellow of the European Society of Cardiology. Her research interests include intravascular imaging, coronary artery disease, and drug eluting stents.

Elazer R. Edelman is an MIT Cabot professor of Health Sciences and Technology, Harvard Medical School professor of Medicine, a senior attending physician in the coronary care unit at the Brigham and Women's Hospital, and director of MIT's Biomedical Engineering Center. His research melds his clinical and research training to study biomaterials, drug delivery, implanted devices, and mechanical support. 